\title{
Guidelines and Post-market Surveillance of Flow Diverter for Intracranial Aneurysms
}

Chiaki Sakai, ${ }^{1}$ Nobuyuki Sakai, ${ }^{2}$ Shigeki Kobayashi, ${ }^{3}$ Koji lihara, ${ }^{4}$ Masayuki Ezura, ${ }^{5}$ and Haruko Yamamoto 6

Flow diverter (FD) is stent-like fine-mesh device for the treatment of intracranial aneurysms, and endovascular therapy with FD has been developed for aneurysms difficult to be cured by standard treatment, and is already widely introduced in other countries. Japanese Society of Neuroendovascular Therapy (JSNET) submitted FD to the government committee as a priority device for the Japanese market in 2011. And, Pipeline Flex (Medtronic Neurovascular, Irvine, CA, USA) was approved in 2015, based on the result of an approving study in Japan and published data of pipeline for uncoilable or failed aneurysms (PUFS) study. At Pipeline Flex approval, three related medical societies, Japan Neurosurgical Society, Japan Stroke Society, and JSNET, established a clinical guideline, including clinical indication of FD, institute criteria, and operator criteria. Furthermore, these societies prepared to operate a clinical database of all cases where FD was used and post-market surveillance will be reported using this database. These activities may work for development of FD in Japan with support of the Ministry of Health, Labour and Welfare, Japan and Pharmaceuticals, and Medical Devices Agency.

Keywords flow diverter, clinical guideline, post-market surveillance

\section{Introduction}

Pipeline Flex (Medtronic Neurovascular, Irvine, CA, USA) obtained approval in 2015 as the first flow diverter (FD), a therapeutic device for difficult-to-treat intracranial aneurysms developed with a new concept, and reimbursement from medical insurance began. Pipeline was designated as a medical device with high therapeutic usefulness by the Ministry of Health, Labour and Welfare (MHLW) committee on

${ }^{1}$ Department of Neurosurgery, Hyogo College of Medicine, Nishinomiya, Hyogo, Japan

${ }^{2}$ Department of Neurosurgery, Kobe City Medical Center General Hospital, Kobe, Hyogo, Japan

${ }^{3}$ Chiba Emergency Medical Center, Chiba, Chiba, Japan

${ }^{4}$ Department of Neurosurgery, Kyushu University Graduate School of Medicine, Fukuoka, Fukuoka, Japan

${ }^{5}$ Department of Neurosurgery, Sendai Medical Center, Sendai, Miyagi, Japan

${ }^{6}$ Center for Advancing Clinical and Translational Sciences, National Cerebral and Cardiovascular Center, Suita, Osaka, Japan

Received: February 29, 2016; Accepted: January 5, 2017 Corresponding author: Nobuyuki Sakai. Department of Neurosurgery, Kobe City Medical Center General Hospital, 2-1-1 Minatojimaminamimachi, Chuo, Kobe, Hyogo 650-0047, Japan

Email: n.sakai@siren.ocn.ne.jp

(C)2017 The Editorial Committee of Journal of Neuroendovascular Therapy. All rights reserved. the priority medical devices to introduction with high clinical needs along with Surpass NeuroEndoGraft (Stryker Neurovascular, Fremont, CA, USA) and gained approval through domestic clinical trials. In its approval, the criteria for its application and guidelines for its proper use were established by related scientific societies similar to medical devices previously approved in this framework, and post-marketing surveillance (PMS) is made obligatory. However, the conditions for the initiation of commercial distribution and clinical use of Pipeline differ widely from medical devices that have been applied to date. In its use, 1) stricter requirements concerning the operator and facility qualifications and training are imposed, and 2) the data of PMS obligated to business firms are provided from the National Inventory Survey Concerning the Efficacy and Safety of Flow Diverters based on industrygovernment-academic collaboration registry led by related scientific societies. In this article, the background and process that resulted in the present state are outlined.

\section{Unapproved Medical Devices with High Clinical Needs}

In 2006, the MHLW initiated a project to have scientific associations recommend unapproved medical devices, the early introduction of which is desired, and to select devices to be introduced through evaluation. ${ }^{1)}$ In the field of neurovascular 
diseases, a carotid artery stent (Precise), an endovascular embolic material (Onyx), an intracranial stent (Wingspan), and vascular embolic beads (Embosphere) have been selected and approved. ${ }^{2)}$ Concerning FDs, the early introduction of Pipeline and Surpass was submitted in September 2011 by the Japanese Society of Neuroendovascular Therapy (JSNET) as the requesting society and the Japan Neurosurgical Society and Japan Stroke Society as related societies, and the FD was listed in February 2012 as "self-expanding implants for the treatment of intracranial aneurysms" at the 18th meeting of the evaluation committee on the early introduction of medical devices with high clinical needs. The committee judged, as FDs are "novel devices, and as perforation of intracranial arteries by the guidewire has occurred overseas, ${ }^{3)}$ it is necessary that the educational system, including the training schemes and institutional criteria for their use, be evaluated primary by the Japanese Society of Neuroendovascular Therapy, which has an board certification system. Of the two devices presently evaluated, i.e., Pipeline and Surpass, the clinical trials of the latter are insufficient in the number of cases and evaluation of the results compared with the former, and further evaluation of clinical results is considered necessary before its introduction." ${ }^{\text {) }}$

Receiving this reply, Covidien (presently Medtronic) initiated a clinical trial to obtain approval in 2012. Details of the trial are described elsewhere, but application for approval of Pipeline was made in September 2014, Pipeline Flex obtained approval in April 2015, and the establishment of institutional criteria (so-called guidelines) became necessary.

\section{Criteria for Application and Guideline for Proper Use}

For the medical devices for which the early introduction has been requested by related scientific societies and that were listed as "medical devices with high clinical needs," the related scientific societies are expected to evaluate the preparation of requirements concerning the system of institution and skills to be acquired by the users, particularly, concerning new medical devices that require particularly high skill levels for use. ${ }^{5)}$ Criteria for application regarding the indications, implementing physicians, and implementing institutions were established concerning the carotid artery stent Precise and distal thrombosis preventive device Angioguard XP in 2008 by 12 related societies in the fields of not only neurovascular but also cardiovascular, radiology, and vascular surgery, concerning the liquid embolic material for embolization before surgical resection for cerebral arteriovenous malformations Onyx in 2008 by four related societies (Japanese Society of Interventional Radiology, Japanese Society of Endovascular Intervention, Japan Neurosurgical Society, and JSNET), and concerning the percutaneous transluminal cerebral thrombectomy device MERCI Retriever in 2010 and the stent for the treatment of intracranial atherosclerosis Wingspan in 2012 by three related societies (Japan Stroke Society, Japan Neurosurgical Society, and JSNET). Concerning the stent for supporting coil embolization of cerebral aneurysms Enterprise VRD in 2010, also, criteria for application were established by three related societies (Japan Stroke Society, Japan Neurosurgical Society, and JSNET).

When Solitaire FR and Trevo ProVue were approved at the end of 2013 and early 2014, respectively, as stent-type thrombus retrieving devices to be used in percutaneous transluminal thrombectomy, three related societies compiled the "Guidelines for the Proper Use of Percutaneous Transluminal Cerebral Thrombectomy Devices (April, 2014)" describing the indications, implementation environment, and points of attention in greater detail, following the "Guidelines for Proper Implementation of Intravenous rt-PA (alteplase) Therapy (October 2005)" compiled by the Japan Stroke Society at the approval of intravenous rt-PA (alteplase) therapy in 2005.6) In the background of these developments were the circumstances in which deployment of stent-type thrombectomy devices became necessary after the efficacy of endovascular recanalization therapy was questioned in the previous year at the International Stroke Conference (ISC). It is still fresh in our memory that this guideline for the proper use was revised after 1 year to the 2nd edition of the "Guidelines for the Proper Use of Percutaneous Transluminal Cerebral Thrombectomy Devices" (April, 2015), following the report supporting the efficacy of endovascular recanalization therapy at the ISC in 2015.

For the approval of FDs, criteria for application have been established by three related societies similar to the previous cases (Table 1). Their contents nearly inherited the previous criteria for application of cerebral artery stenting to support coil embolization, but a striking difference was that the implementing facilities were prompted to register all patients treated by the use of FDs. It was against this background that the "National Inventory Survey Concerning the Efficacy and Safety of Flow Diverters," which is explained later, was organized and put into effect. Also, guidelines for the proper use were established by three related societies similar to "devices for percutaneous transluminal thrombectomy", and, for their 
Table 1 The Japan Neurosurgical Society, The Japan Stroke Society, and The Japanese Society for Neuroendovascular Therapy

Criteria for application of intracranial arterial stents (Flow diverter for the treatment of cerebral aneurysms) (April, 2015)

[Indications]

Cerebral aneurysms

Indications of individual devices are based on the conditions of pharmaceutical approval of each device.

[Criteria for implementing facilities]

Equipment: The operation room or angiography room must be equipped with an appropriate angiography system.

Treatment environment: The facilities must have an environment in which neurosurgery can always be performed promptly.

Patient registration: The facilities must participate in a survey of patients undergoing the treatment and register all patients.

[Criteria for implementing physicians]

Qualifications certified by the scientific society: The physicians must be experts in cerebral endovascular therapy with sufficient experience in endovascular treatment for cerebral aneurysms.

Required training: The physicians must finish the training program of the medical device in question.

[Supplementary items]

Review system: The facilities and physicians must participate in the post-marketing survey (PMS).

The criteria for application will be revised after three years, in principle, on the basis of the results of the post-marketing survey.

establishment, also, public invitation to the project to formulate standards including requirements for the use of new medical devices (stents for the treatment of cerebral aneurysms) $)^{7)}$ was made, and the JSNET, which requested designation of the FD as a medical device with high clinical needs, applied and established the "Guidelines for the Proper Use of Intracranial Arterial Stents (Flow Diverters for the Treatment of Cerebral Aneurysms) (March 2013)." The guidelines were revised to the 2nd edition (April 2015) simultaneously with the approval of Pipeline Flex in April 2015 and have since been in effect (Table 2). I understand that this project was carried out as the administrative authorities acknowledged the role played by scientific societies in the deployment of novel drugs and medical devices.

\section{Post-market Surveillance}

As mentioned above, concerning devices that are novel, permanently implanted, and highly invasive, such as Pipeline, it is important to collect and evaluate data concerning the efficacy and safety including those on long-term use after marketing and feed them back to the business firms and administration. PMS used to be carried out for a period as part of the review system of novel medical devices as an obligation imposed on the manufacturers. However, industry-operated PMS has been suggested to have the following problems: 1) The data are not obtained by strict planning or monitoring as in clinical trials, 2) the medical workers, who are expected to provide data, are poorly motivated due to the sense of burden and lack of incentives for data registration, and 3) long-term data collection is difficult. For these reasons, despite the heavy economic burden imposed on the business firms, trust from the administration was low, the possibility of concomitant use of the product with those of other companies or its off-label use was small, and, consequently, the survey was at the risk of deviation from the real world. Post-marketing registry conducted with industry-government-academic cooperation is attracting attention as an effective solution to these problems. ${ }^{8)}$

In Japan, also, following the examples of foreign countries, two registries, that is, 1) the Japanese Registry for Mechanically Assisted Circulatory Support (J-MACS) using implantable ventricular assist devices (VAS) was started in 2010, and 2) registry of patients undergoing transcatheter aortic valve implantation (TAVI registry) was started in 2013. Concerning Pipeline, the "Flow Diverter Database Management Committee" consisting of a total of 10 members from the Japan Neurosurgical Society, Japanese Society for Neuroendovascular Therapy, and Japan Stroke Society was established in July 2015, and a study using an electronic information registration system of the National Clinical Database Center (NCD) was initiated as the "National Inventory Survey Concerning the Efficacy and Safety of Flow Diverters" (Fig. 1). In this project, PMS is performed by planning under the leadership of scientific societies, and part of the results (120 cases) are provided to business firms as PMS data. This is the third of the evaluations of the results of the use of FDs in this form, and by the use of the registry in which academia is positively involved, the compelling force of the academia and quality of the data derived from the initiative of a scientific society are expected to be guaranteed, and the economic burden of business firms to be reduced.

\section{Discussion}

Endovascular treatment for intracranial aneurysms using FDs was started with great expectations as an epoch-making treatment for lesions difficult to treat by conventional therapies. For the JSNET, serving as the secretariat, to formulate criteria for application and guidelines for proper use of FDs 
Table 2 Second edition of the guidelines for the proper use of intracranial arterial stents (Flow diverter for the treatment of cerebra aneurysms) (April, 2015) (excerpts)

The Japan Neurosurgical Society, The Japan Stroke Society, and The Japanese Society for Neuroendovascular Therapy

Recommendation notes

Device

1. An FD that has obtained pharmaceutical approval must be used for the treatment.

Presently, Pipeline (Covidien/Medtronic) is the only FD that has obtained pharmaceutical approval, but Surpass (Stryker) and FRED (Terumo/Microvention) are undergoing clinical trials. There are products of other companies including Silk (BALT), Bravo (Codman/Johnson\&Johnson), and MFM (Cardiatis) that are already in clinical use and are planned to be introduced to Japan. Indications

2. The indications are based on the conditions of pharmaceutical approval of individual medical devices.

Pipeline is indicated for unruptured cerebral aneurysms with a maximum diameter of $10 \mathrm{~mm}$ or above located in the internal carotid artery proximal to the origin of the posterior communicating artery, and its application to other regions is not approved. Also, it must be applied carefully to lesions considered to be safely and curatively treated by conventional surgical or endovascular procedures.

For reference, the clinical trial of Surpass is carried out against unruptured cerebral aneurysms with a maximum diameter of $10 \mathrm{~mm}$ or above located in the internal carotid artery except the terminal portion, and the clinical trial of FRED is carried out against lesions including saccular, fusiform, and multilocular aneurysms $10 \mathrm{~mm}$ or greater in diameter located in the internal carotid, vertebral, or basilar artery.

3. The implementing medical organizations must have an environment equipped with a high-performance angiography system in which neurosurgery can always be performed.

It is essential that the implementing facility has an environment in which endovascular treatment of the brain, that is, an angiography room or operation room equipped with an angiography system. In addition, since surgical treatment may be necessary during or after the treatment, it is also necessary to have an environment in which neurosurgery can always be performed. For safe placement of the device, its visibility under X-ray fluoroscopy is important, and it is desirable that the facility is equipped with a high-performance angiography system.

4. The implementing physicians must be experts in endovascular treatment of the brain with experience in endovascular treatment of the brain, particularly endovascular treatment for cerebral aneurysms, and have sufficient experience in stent-assisted embolization of cerebral aneurysms.

Experience in endoscopic treatment for cerebral aneurysms, particularly stent-assisted embolization of cerebral aneurysms, is useful for the actual procedure of FD placement and follow-up of patients from before and after the procedure. Treatment

5. Angiography is performed preoperatively, and the accurate vascular diameter and deployment length are measured. An appropriate device is selected in consideration of the shape of the cerebral aneurysm and vascular arrangement. MRI may be useful for the prediction of hemorrhagic complications.

6. FDs must be placed safely and accurately by observing the standard handling and deployment methods of each device.

7. The apposition of the FD to the parent vessel is confirmed after the placement. Antithrombotic therapy.

8. Concomitant antiplatelet therapy is recommended to be performed from the perioperative to postoperative period.

9. It is recommended to preoperatively confirm factors that prevent the implementation of continuous antithrombotic therapy.

10. Appropriate management of postoperative hemorrhagic complications is required. Other treatments

11. While the natural history of cerebral aneurysm, considered to be an indication for FDs, is not clear, it must be noted that there are situations in which conservative follow-up should be selected.

12. It must be noted that there are situations in which surgery or other endovascular procedures can be relatively safe and effective depending on the shape or site of the cerebral aneurysm, state of the collateral blood flow, and previous intracranial or extracranial bypass surgery.

FD: Flow diverter

along with the Japan Neurosurgical Society and Japan Stroke Society is a requirement for obtaining approval, and compliance to them is strongly demanded in the treatment after approval. Moreover, as performing PMS to validate the safety and therapeutic efficacy is also a condition for approval, it was decided that PMS of FDs be carried out in a new framework constituted by academia-industry collaboration in which the Japan Neurosurgical Society plays the leading role. Partly because most of the medical devices used in Japan were developed overseas, many of us neuroendovascular therapists have completely relied on business firms and administrative authorities in the development and improvement of medical devices. However, to develop and improve medical devices useful and safe for neuroendovascular treatments in Japan, it is indispensable for ourselves to collect and analyze highly reliable high-quality data concerning the results and real state of the use of FDs and feed them back to business firms and the administration. The present "National Inventory Survey on the Efficacy and Safety of Flow Diverters" is the first registry in the field of neurovascular diseases performed by industry-government-academia collaboration, and it is expected to exert a considerable impact on improvements 


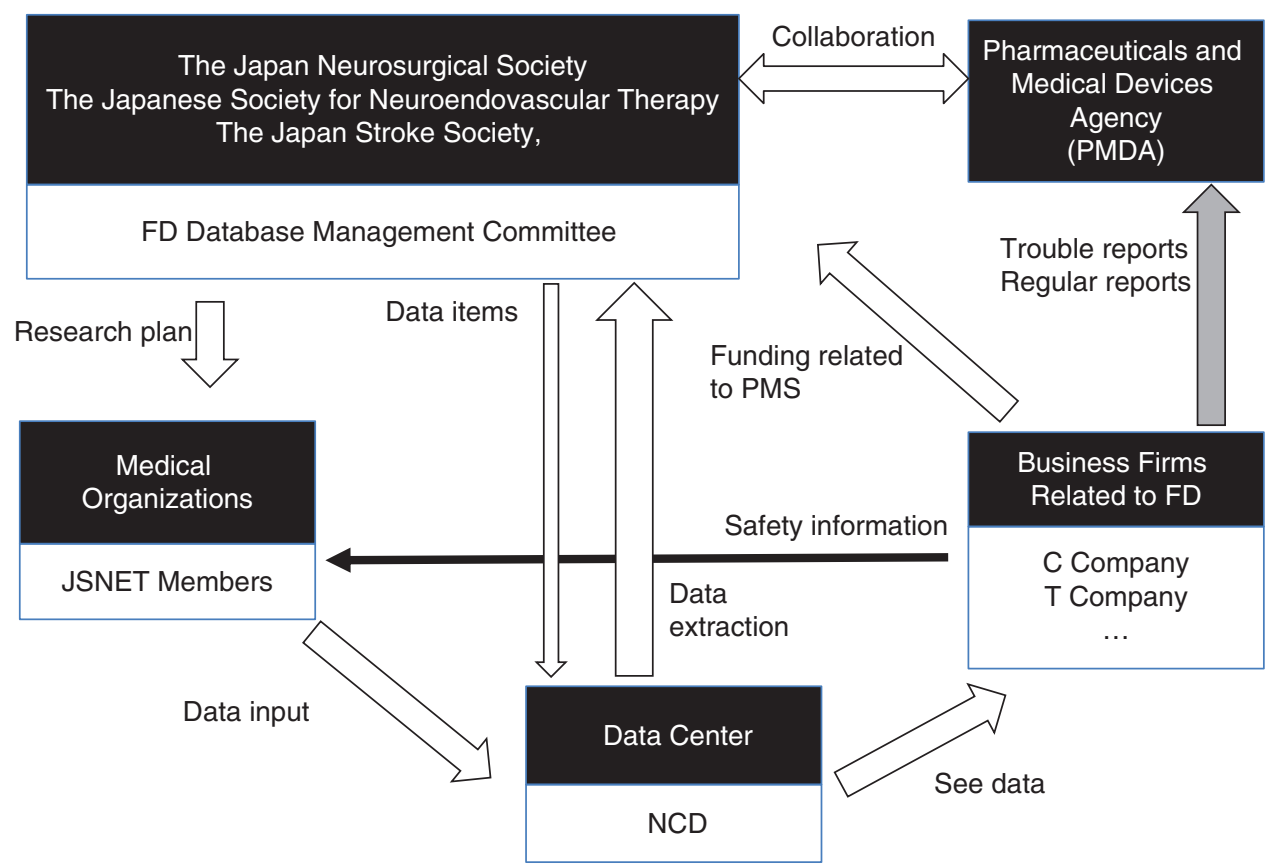

Fig. 1 Parties participating in the national inventory survey concerning the efficacy and safety of flow diverters and their roles. FD: Flow diverter; JSNET: The Japanese Society for Neuroendovascular Therapy; PMS: post-marketing survey; NCD: National Clinical Database Center

and development of FDs and modification of their indications. To secure such benefits from the registry, active participation of users is essential. The objective of having novel medical devices, including FDs, properly developed in Japan is expected to be achieved through many of the approaches mentioned in this article.

\section{Conclusion}

FDs are novel medical devices that markedly increase the potential of endovascular treatment for intracranial aneurysms, and, for their development, related scientific societies formulated criteria for application and guidelines for proper use while being advised and supported by the administration. Also, academia-operated registry study is planned, and the data are expected to be used for post-marketing reviews. Through industry-government-academia collaboration, FDs will be introduced safely in Japan, and their effectiveness to be validated.

\section{Acknowledgments}

Members of the Committee for the establishment of Criteria for Application and Guidelines for the Proper Use of Intracranial Arterial Stents (Flow Diverters for the Treatment of Cerebral Aneurysms), 2012

\section{The Japan Neurosurgical Society}

Isao Date, (Medical Devices Committee) Department of Neurological Surgery, Okayama University

Nobuto Saito, (Scientific Committee) Department of Neurosurgery, The University of Tokyo

Yoshiaki Shiokawa, (Insurance Committee) Department of Neurosurgery, Kyorin University

\section{The Japan Stroke Society}

Kazuo Minematsu, National Cerebral and Cardiovascular Center

Kuniaki Ogasawara, Department of Neurosurgery, Iwate Medical University

Masahiro Yasaka, Department of Cerebrovascular Medicine and Neurology, Clinical Research Institute, National Hospital Organization, Kyushu Medical Center

\section{The Japanese Society for Neuroendovascular Therapy}

Akira Ishi, Department of Neurosurgery, Kyoto University Shigeru Miyachi, Department of Endovascular Surgery, Osaka Medical College

Shigeru Nemoto, Department of Endovascular Surgery, Tokyo Medical and Dental University

Nobuyuki Sakai, Department of Neurosurgery, Kobe City Medical Center General Hospital: General affairs 
Members of the Committee for the Formulation of Criteria for Application and Guidelines for the Proper Use of Intracranial Arterial Stents, 2nd Edition (Flow Diverters for the Treatment of Cerebral Aneurysms), 2015

\section{The Japan Neurosurgical Society}

Koji Iihara, Department of Neurosurgery, Kyushu University

Kenji Ohata, Department of Neurosurgery, Osaka City University

Nobutaka Kawahara, Department of Neurosurgery, Yokohama City University

\section{The Japan Stroke Society}

Kuniaki Ogasawara, Department of Neurosurgery, Iwate Medical University

Kazuo Minematsu, National Cerebral and Cardiovascular Center

Masahiro Yasaka, Department of Cerebrovascular Medicine and Neurology, Clinical Research Institute, National Hospital Organization, Kyushu Medical Center

\section{The Japanese Society for Neuroendovascular Therapy}

Akira Ishii, Department of Neurosurgery, Kyoto University

Shigeru Nemoto, Department of Endovascular Surgery, Tokyo Medical and Dental University

Shigeru Miyachi, Department of Endovascular Surgery, Osaka Medical College

Nobuyuki Sakai, Department of Neurosurgery, Kobe City Medical Center General Hospital (Clerical office)

Flow Diverter Database Management Committee, 2015

\section{The Japan Neurosurgical Society}

Kenji Ohata, Department of Neurosurgery, Osaka City University (Chairman)

Masayuki Ezura, Department of Neurosurgery, Sendai Medical Center

Hiroyuki Kinouchi, Department of Neurosurgery, University of Yamanashi

Yuichi Murayama, Department of Neurosurgery, Jikei University School of Medicine

Naoki Nakayama, Department of Neurosurgery, Hokkaido University

Yuhei Yoshimoto, Department of Neurosurgery, Gunma University

\section{The Japan Stroke Society}

Kazuo Minematsu, National Cerebral and Cardiovascular Center

Masahiro Yasaka, Department of Cerebrovascular Medicine and Neurology, Clinical Research Institute, National Hospital Organization, Kyushu Medical Center

\section{The Japanese Society for Neuroendovascular Therapy}

Koji Iihara, Department of Neurosurgery, Kyushu University

Shigeki Kobayashi, Department of Neurosurgery, Chiba Emergency Medical Center

Nobuyuki Sakai, Department of Neurosurgery, Kobe City Medical Center General Hospital

\section{Disclosure Statement}

The top and corresponding authors have completed selfreporting of conflict of interest (COI) to the Japan Neurosurgical Society. The COI that the corresponding author should disclose in connection with this article is that he was paid an annual sum of one million yen or less by Medtronic Japan Co., Ltd. for the time and effort needed for attending (making presentations at) conferences. No other authors had COI.

\section{References}

1) MHLW committee for the evaluation of early introduction of medical devices with high clinical needs. (in Japanese) http:// www.mhlw.go.jp/stf/shingi/other-iyaku.html?tid=128705. (accessed February, 29, 2016)

2) Handout 1 for the 18th meeting of the committee for the evaluation of early introduction of medical devices with high clinical needs. (in Japanese) http://www.mhlw.go.jp/ stf/shingi/2r98520000021 zxd-att/2r98520000021zyu.pdf. (accessed February, 29, 2016)

3) Jabbour P, Chalouhi N, Tjoumakaris S, et al: The pipeline embolization device: learning curve and predictors of complications and aneurysm obliteration. Neurosurgery 2013; 73: 113-120; discussion 120.

4) Handout 4-2 for the 18th meeting of the committee for the evaluation of early introduction of medical devices with high clinical needs. (in Japanese) http://www.mhlw.go.jp/ stf/shingi/2r98520000021zxd-att/2r98520000022031.pdf. (accessed February, 29, 2016)

5) Handout 8 for the 3rd meeting of the committee for the evaluation of early introduction of medical devices with high clinical needs. (in Japanese) http://www.mhlw.go.jp/ 
shingi/2007/06/dl/s0607-10h.pdf. (accessed February, 29, 2016)

6) Intravenous rt-PA (alteplase) therapy guidelines subcommittee, Medical care improvement/social insurance committee, Japan Stroke Society: Guidelines for proper implementation of intravenous rt-PA (alteplase) therapy (October, 2005). Stroke 2005; 27: 327-354. (in Japanese)
7) Project for the formulation of criteria including requirements for the use of new medical devices (stent for the treatment of cerebral aneurysms). (in Japanese) http:// www.mhlw.go.jp/sinsei/chotatu/chotatu/kikaku/2012/06/ kk0601-02.html. (accessed February, 29, 2016)

8) Ishii K, Tawaragi T: Post-marketing safety measures for medical devices: PMS and registry. Regulatory Science of Medical Products 2015; 5: 73-80. (in Japanese) 